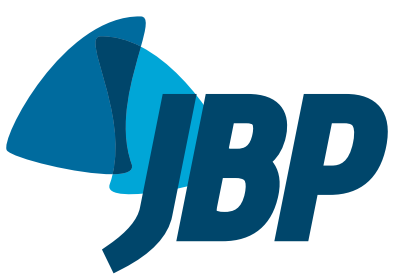

\section{Tree-in-bud pattern}

\author{
Edson Marchiori¹, Bruno Hochhegger², Gláucia Zanetti ${ }^{1}$
}

A 34-year-old female patient presented to the outpatient clinic with complaints of fever, cough, and weight loss ( $8 \mathrm{~kg}$ in the last 3 months). She had a history of having undergone resection of a uterine adenocarcinoma 2 years prior. A chest CT showed branching opacities, which characterize the tree-in-bud (TIB) pattern, in the middle lobe and the left lower lobe, as well as left lung volume reduction and consolidation with cavitation in the lingula (Figure 1).

The TIB pattern represents centrilobular branching opacities, most pronounced in the lung periphery, resembling the budding of certain plants. Although, in most cases, the TIB pattern represents filling of bronchioles with mucus, pus, blood, or other materials, with or without dilatation, it may also be related to peribronchovascular connective tissue thickening or filling and dilatation of pulmonary arterioles.

Although the TIB pattern is more commonly observed in bronchiolar infections (bronchopneumonia, infectious bronchiolitis, and endobronchial dissemination of tuberculosis), it has a broader differential diagnosis that includes bacterial infections, fungal infections (allergic bronchopulmonary aspergillosis), or viral infections; as well as bronchiectasis; idiopathic conditions (obliterative bronchiolitis and panbronchiolitis) or congenital conditions (cystic fibrosis); aspiration or inhalation of foreign material; and peripheral pulmonary vascular disease, especially due to endovascular metastatic spread of certain tumors.
In our patient, the main differential diagnosis was between pulmonary tuberculosis and endovascular metastatic spread of the previously resected uterine tumor (neoplastic embolism).

Pulmonary tumor embolism is the occlusion of pulmonary arteries and arterioles by spreading neoplastic cells. It occurs more frequently in patients with lung, gastrointestinal, liver, breast, or uterine cancer. The possibility of tumor embolism should be considered when a patient with known malignancy develops dyspnea. A suggestive CT finding of neoplastic embolism is dilatation of the affected vessels due to growth of the tumor emboli, a finding that was not observed in our patient.

In tuberculosis, the finding of a TIB pattern is a strong indication of disease activity, as well as of the presence of cavitation. A concomitant finding of adjacent bronchial wall thickening is also a strong indication of bronchial inflammation, suggesting an infectious process. In our patient, sputum smear microscopy was positive for AFB, and culture revealed Mycobacterium tuberculosis. The final diagnosis was pulmonary tuberculosis.

Radiologists and pulmonologists need to be aware of the broad range of etiologies for the TIB pattern in order to properly guide further investigation. A combination of patient history, clinical and laboratory findings, and associated CT findings is usually sufficient to reach a final diagnosis.
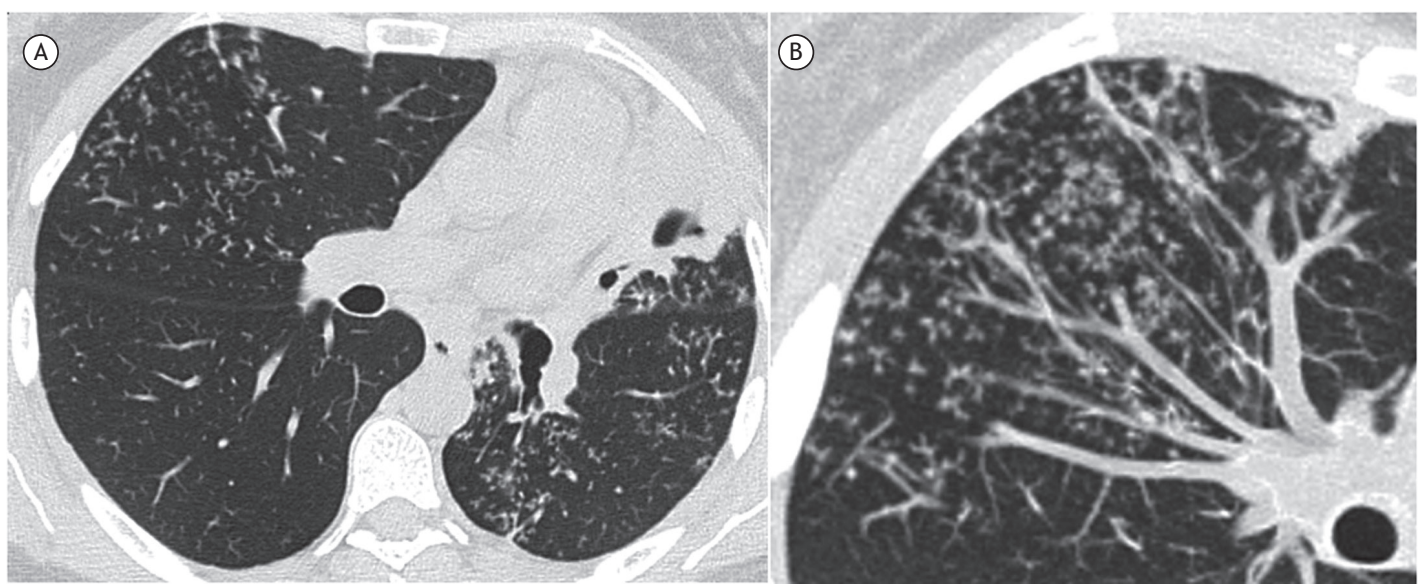

Figure 1. In A, axial CT scan of the chest with lung window settings at the level of the subcarinal region, showing left lung volume reduction and consolidation with cavitation in the lingula. Note also centrilobular branching opacities (tree-in-bud pattern) in the middle lobe and the left lower lobe. In B, image enhancement (maximum intensity projection) demonstrating the tree-in-bud pattern.

\title{
RECOMMENDED READING
}

Fraser RS, Müller NL, Colman NC, Pare PD, editors. Diagnosis of Diseases of the Chest. 4th ed. Philadelphia: WB Saunders Company; 1999. 